\title{
Postmortem CT pulmonary findings in SARS-CoV-2-positive cases: correlation with lung histopathological findings and autopsy results
}

\author{
Laura Filograna ${ }^{1}$ - Simone Grassi ${ }^{2}$. Guglielmo Manenti ${ }^{1} \cdot$ Carlo Di Donna $^{1} \cdot$ Doriana Tatulli ${ }^{1} \cdot$ Francesco Nardoni $^{2}$. \\ Valentina Masini ${ }^{3} \cdot$ Francesco Ausania $^{2}$. Vincenzo Maria Grassi ${ }^{2} \cdot$ Roberto Floris $^{1} \cdot$ Cesare Colosimo ${ }^{3}$. \\ Vincenzo Arena ${ }^{4} \cdot$ Vincenzo Lorenzo Pascali $^{2} \cdot$ Antonio Oliva $^{2}$
}

Received: 23 July 2021 / Accepted: 26 January 2022 / Published online: 14 February 2022

(c) The Author(s), under exclusive licence to Springer-Verlag GmbH Germany, part of Springer Nature 2022

\begin{abstract}
Introduction/purpose Postmortem computed tomography (PMCT) is a valuable tool for analyzing the death of patients with SARS-CoV-2 infection. The purpose of this study was to investigate the correlation between PMCT lung findings in autopsy cadavers positive for SARS-CoV-2 infection and the severity of COVID-19 lung disease by histopathological analysis.

Materials and methods We reviewed chest PMCT findings, paying particular attention to the lung parenchyma, in 8 autopsy cases positive for SARS-CoV-2. Correlations between chest PMCT and histopathological findings were assessed. Clinical conditions and comorbidities were also recorded and discussed. The primary cause of death was finally considered.

Results In 6/8 cases, pulmonary PMCT findings were massive consolidation (4/8) and bilateral diffuse mixed densities with a crazy-paving pattern (2/8). These cases showed severe pulmonary signs of COVID-19 at histopathological analysis. In the remaining 2/8 cases, pulmonary PMCT findings were scant antideclive ground-glass opacities in prevalent gradient densities attributed to hypostasis. In $4 / 8$ cases with massive consolidations, important comorbidities were noted. In $6 / 8$ cases with severe pulmonary histopathological signs of lung COVID-19, autopsy found that the cause of death was cardiorespiratory failure. In the remaining $2 / 8$ cases, histopathological analysis revealed lung alterations due to edema and some signs of SARS-CoV-2 infection; the cause of death was not attributed to SARS-CoV-2 infection (Table 1).

Discussion and conclusion Chest PMCT findings correlate with the severity of COVID-19 lung disease at histopathology examination. According to our results, there may also be a relationship between cause of death and PMCT findings in COVID-19, which must be critically analyzed considering clinical antemortem data.
\end{abstract}

Keywords Postmortem CT · COVID-19 $\cdot$ SARS-CoV-2 $\cdot$ Lung findings $\cdot$ Pulmonary histopathology

\section{Introduction}

COVID-19 causes a multiorgan disease, with clinical manifestations ranging from asymptomatic to multiple organ dysfunction syndrome (MODS). The most common clinical

Laura Filograna and Simone Grassi are co-first authors.

Laura Filograna

laura.filograna@gmail.com

1 Department of Integrated Care Processes, Diagnostic Imaging Area, Tor Vergata University, PTV Policlinico Tor Vergata, Viale Oxford 81, 00133 FondazioneRome, Italy

2 Department of Health Surveillance and Bioethics, Section of Legal Medicine, Catholic University of Sacred Heart, Fondazione Policlinico Universitario A. Gemelli IRCCS, Largo Francesco Vito, 1, 00168 Rome, Italy manifestations are respiratory symptoms, ranging from mild upper-respiratory symptoms to acute respiratory distress syndrome (ARDS) [1]. Although COVID-19 is usually characterized by a multiorgan effect associated with hyperactivation of the immune system response, it mainly affects the lungs. Several studies based on autopsy cases have explored

3 Department of Diagnostic Imaging, Oncological Radiotherapy and Hematology - Diagnostic Imaging Area, Catholic University of Sacred Heart, Fondazione Policlinico Universitario Agostino Gemelli IRCCS, Largo Francesco Vito, 1, 00168 Rome, Italy

4 Department of Woman and Child Health and Public Health, Area of Pathology, Catholic University of Sacred Heart, Fondazione Policlinico Universitario A. Gemelli IRCCS, Largo Francesco Vito, 1, 00168 Rome, Italy 


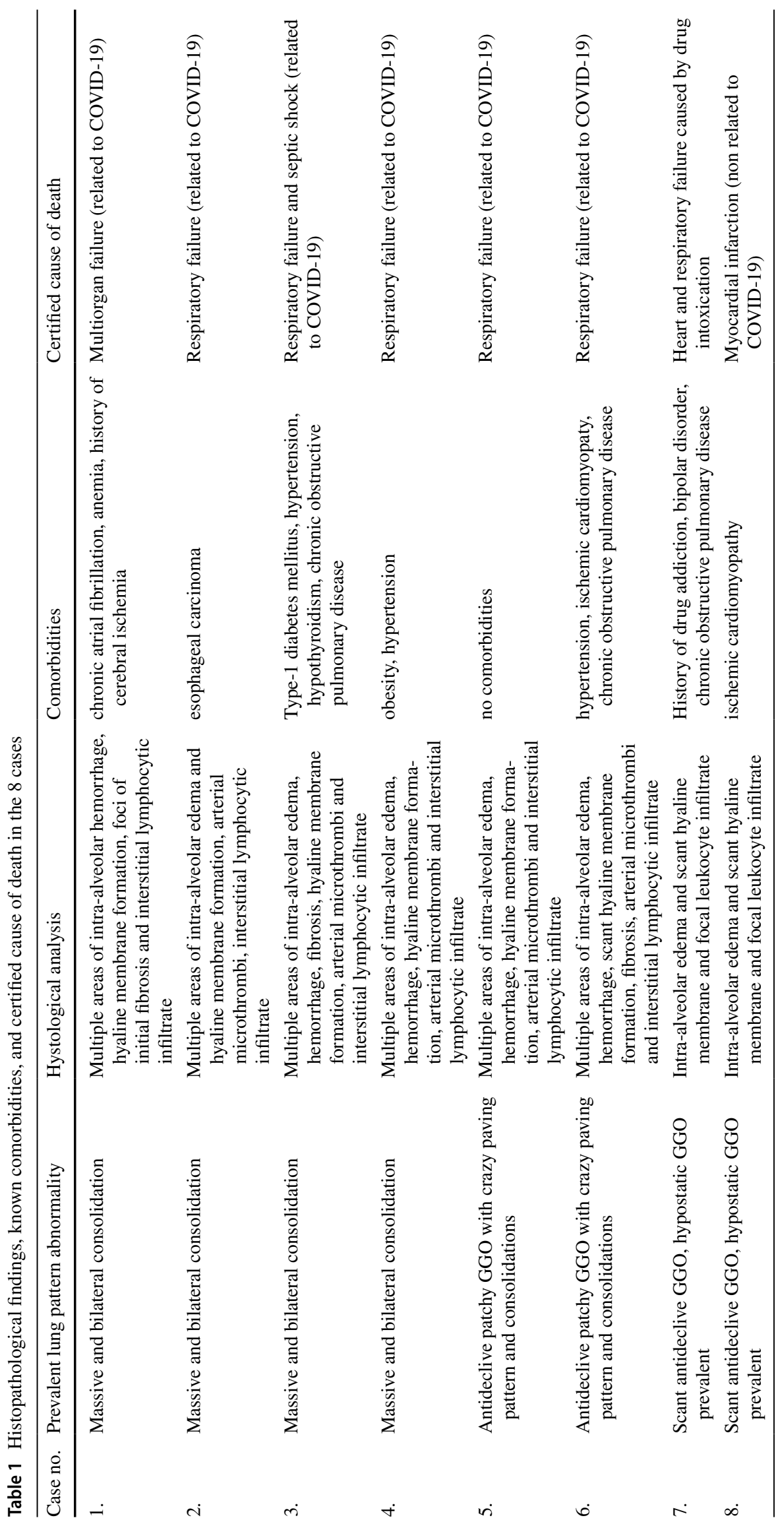


histopathological findings in SARS-CoV-2 infection [2-5]. The main histopathological features of the lungs include diffuse alveolar damage with the development of hyaline membranes, macrophage activation in air spaces, and thickening of the alveolar wall $[5,6]$. Since the beginning of the pandemic, $\mathrm{X}$-rays and/or chest computed tomography (CT) have been proposed as primary diagnostic tools for COVID-19 cases. Indeed, Kim et al. performed a meta-analysis of 68 studies, finding that imaging techniques can be even more sensitive than microbiological tests (in particular, the sensitivity of chest CT, at 94\%, was found to be higher than that of real-time polymerase chain reaction (RTPCR), at 89\%) [7]. Different CT patterns of COVID-19 pulmonary affection have been reported [8-15]. The most typical pulmonary CT findings in COVID19 patients according to Karimian et al. [14] are bilateral lung involvement by ground-glass opacity (GGO) or mixed (GGO pulse consolidation or reticular) patterns, thickened interlobular septa, vascular enlargement, air bronchogram, peripheral distribution, and left and right lower lobe involvement. Complete consolidation of both lungs (indicative of ARDS) has also been reported in severe and advanced pulmonary COVID19 [14]. In addition to the clinical literature, some postmortem CT (PMCT) imaging findings have been reported, though they are not specific or highly suggestive of lung SARS-CoV-2 infection [16-24]. As a consequence, PMCT imaging has been proposed as a useful tool for postmortem investigations in diagnosed or suspected cases of SARS-CoV-2 infection to augment the numbers of postmortem examinations, especially in the first wave [25] or more recently as a screening method for SARS-CoV-2 infection before autopsy [16-22, 24]. In fact, due to the importance of preautopsy assessment of biological risk and the complexity of properly examining COVID-19 multiorgan involvement through sole autopsy, PMCT has been widely adopted in forensic investigations of SARS-CoV-2 suspected or ascertained-related deaths [16-24], especially during the first phase of the pandemic.

Nevertheless, both to validate PMCT in COVID-19 cases and ensure its diagnostic value - particularly when an autopsy is not performed-it is important to find evidence regarding concordance between data obtained through PMCT and through traditional forensic autopsy. Therefore, in this retrospective study, we compared PMCT lung findings and the presence and severity of pneumonia at histopathological analysis in forensic cases of COVID-19. A further aim was to investigate correlation between PMCT lung findings in SARSCoV-2-positive cadavers and cause of death (Table 1).

\section{Material and methods}

A retrospective study was designed by considering a study population selected through the application of three inclusion criteria and two exclusion criteria. The inclusion criteria were (i) death during hospitalization (period: March 2020-January 2021) with microbiological evidence of SARS-CoV-2 infection and clinical diagnosis of COVID19 , (ii) age older than 18 , and (iii) postmortem radiological data obtained through PMCT. The exclusion criteria were (i) full forensic autopsy not performed and (ii) presence of macroscopic/microscopic/radiological signs of advanced putrefaction (postmortem interval higher than $72 \mathrm{~h}$ ). Time since death was always known because it was reported by clinical documentation. Postmortem intervals higher than $72 \mathrm{~h}$ were not considered since, as reported by Egger et al., in these late stages, postmortem changes (and, in particular, the presence of gas inside the organs) are particularly relevant and thus can jeopardize a correct radiological evaluation [26]. Wholebody PMCT examination was performed using a 16-slice scanner (Philips Medical Systems, Best, The Netherlands) with the following parameters: slice acquisition $1.25 \mathrm{~mm}$, pitch 0.5 , rotation time $0.5 \mathrm{~s}$, tube voltage $120 \mathrm{kVp}$, tube current-time $400 \mathrm{mAs} /$ rotation. No contrast medium was administered. Image reconstruction was carried out at a slice thickness of $1 \mathrm{~mm}$ (0.6-mm increment), with soft tissue and sharp bone kernel. Lung parenchyma alterations on PMCT imaging were annotated. A full forensic autopsy comprehensive histopathological examination was performed for each case. Correlations between lung PMCT and histopathological findings with particular attention to those suggestive of lung SARS-CoV-2 infection were assessed. Data regarding clinical conditions/comorbidities were also collected, and the primary cause of death was considered.

\section{Results}

\section{Study population}

The final study population consisted of 8 cases, 5 males and 3 females, whose ages ranged from 36 to 89 years (mean age: 65 years). With the exception of case 5 (a 47 -year-old man), all subjects had at least one comorbidity: heart disease (3/8 37,5\%), obesity (1/8 12,5\%), hypertension ( $3 / 837,5 \%)$, oncological pathology (1/8 12,5\%), lung disease (COPD 3/8 $37,5 \%)$, diabetes mellitus type $1(1 / 812,5 \%)$, drug abuse $(1 / 812,5)$, cerebral ischemia $(1 / 812,5 \%)$, and hypothyroidism $(1 / 812,5 \%)$. All patients received oxigenotherapy and invasive or noninvasive mechanical ventilation for at least 2 days. No patients were vaccinated, as the national vaccination campaign had not been started yet in our country in the study period (March 2020-January 2021).

\section{Pulmonary PMCT imaging analysis}

In 4/8 cases, massive and bilateral consolidations involving the majority of both lungs were observed (and classified 
as pattern 1) (Fig. 1). In $2 / 8$ cases, bilateral and multifocal ground-glass opacities with internal reticular inter- and intralobular septal thickening ("crazy-paving pattern") and consolidations both with peripheral/subpleural predilection were found (and classified as pattern 2) (Fig. 2). In the last $2 / 8$ cases, some antideclive areas of GGO in the context of prevalent gradient GGO in both lungs attributed mainly to hypostasis were found (and classified as pattern 3) (Fig. 3).

\section{Comparison between PMCT and autopsy findings}

In 6/8 cases with patterns 1 and 2 (case nos. 1, 2, 3, 4, 5, 6), histopathological analysis revealed severe pulmonary coronavirus disease represented by different degrees of edema, hyaline membranes, intra-alveolar hemorrhage, arterial microthrombi, foci of initial fibrosis and interstitial lymphocytic infiltrate (Fig. 4). These features were more severe in cases of pattern 1 . In the remaining $2 / 8$ cases with pattern 3 according to PMCT, histopathological analysis revealed lung alterations due to edema and some signs of SARS-CoV-2 infection represented by scant hyaline membrane and focal leukocyte infiltrate (Fig. 5).

\section{Correlation with cause of death}

For the $6 / 8$ cases with patterns 1 and 2, the autopsy-ascertained cause of death was cardiorespiratory failure due to SARS-CoV-2 infection. In the 2 cases of pattern 3, the cause of death was ischemic heart failure in subjects with initial lung COVID-19. COVID-19 was indicated (in the $6 / 8$ cases) as the cause of the death combining clinical, radiological, macroscopic, and microscopic findings and excluding alternative causes at the forensic examination (as an ischemic heart failure, certified as the cause of the death in the remaining cases).

\section{Discussion}

The potential of PMCT in detecting pulmonary alterations significant for postmortem analysis has been reported before [27-29]. In the forensic field, PMCT imaging of the lungs is often used to guide autopsy and enhance diagnostic sensitivity, even when an autopsy cannot be performed [30]. Overall, lung CT is an excellent tool for both clinical and postmortem investigations because of the natural contrast caused by air. After death, possible bias is represented by postmortem changes, particularly by hypostases that usually appear as GGOs with a frank and declive distribution [27], in opposition to the GGOs of SARS-CoV-2 that are generally patchy and scattered. In particular, as shown herein, PMCT for cadavers with positive nasopharyngeal and oral swabs showed alterations that can be differentiated by hypostases. These alterations were represented by massive and bilateral consolidations involving the majority of both lungs, identified as pattern 1 (case nos. 1, 2, 3, 4), bilateral and multifocal ground-glass opacities with a "crazy-paving pattern" and consolidations both with peripheral/subpleural predilection classified as pattern 2 (case nos. 5 and 6), and a few antideclive areas of GGO in the context of hypostatic GGO in both lungs classified as pattern 3 (case nos. 7 and 8). Lung PMCT patterns 1 and 2 identified in our study population are concordant with the most common findings on PMCT reported in the literature as the expression of severe pulmonary COVID-19 [16-24], though a higher prevalence of massive consolidation and GGOs of both lungs (pattern

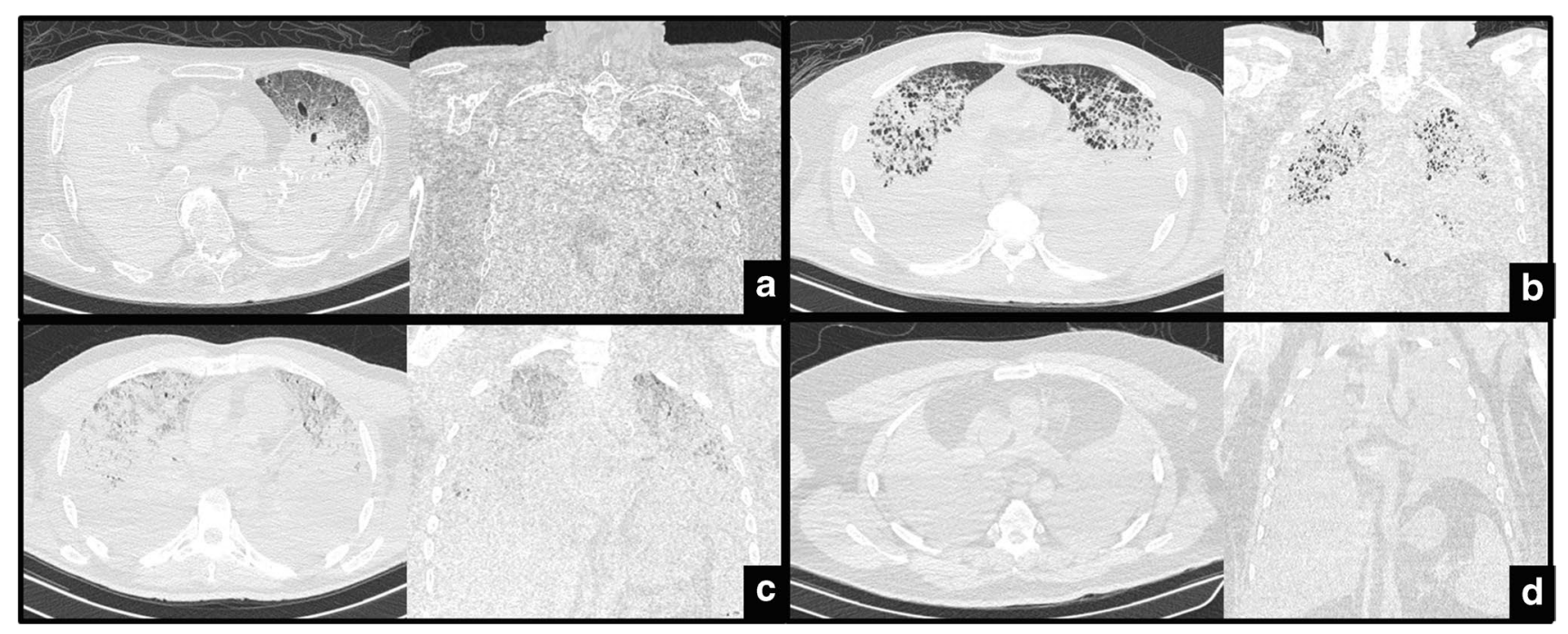

Fig. 1 Axial (left) and coronal (right) PMCT images at the level of the thorax, with lung window) in case nos. 1 (a), 2 (b), 3 (c), and 4 (d) with pulmonary PMCT pattern 1 . Note that all cases show massive and bilateral consolidations involving the majority of both lungs. 
Fig. 2 Axial (left) and coronal (right) PMCT images at the level of the thorax, with lung window, in case nos. 5 (a) and 6 (b), with pulmonary PMCT pattern 2 . Note that all cases show bilateral and multifocal groundglass opacities with internal reticular inter- and intralobular septal thickening ("crazy-paving pattern") (arrows exemplar) and consolidations (arrowheads exemplar), both with peripheral/ subpleural predilection.
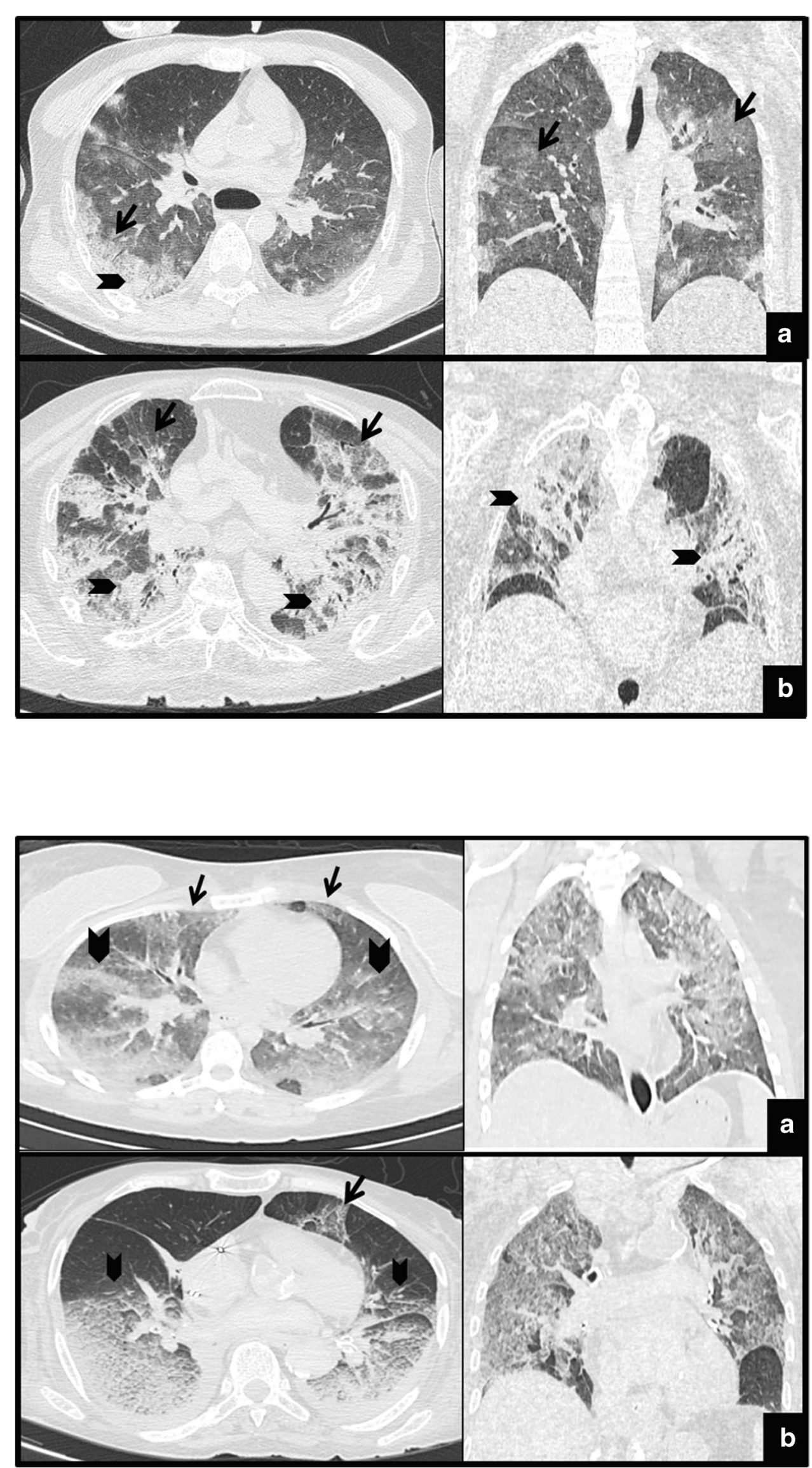

Fig. 3 Axial (left) and coronal (right) PMCT images at the level of the thorax, with the lung window, in case nos. 7 (a) and 8 (b), with pulmonary PMCT pattern 3. Note few antideclive areas of GGOs (arrows exemplar) in the context of prevalent gradient GGOs in both lungs attributed mainly to hypostasis (arrowheads). Image (b) shows the probable copresence of hypostasis and edema. 
Fig. 4 Histological findings associated with PMCT patterns 1 and 2: (a and b) alveolar damage with hyaline membrane (hematoxylin and eosin staining, $5 \times$ magnification); (c) foci of initial fibrosis and interstitial lymphocytic infiltrate (hematoxylin and eosin staining, 10x magnification); (d) oedema, interstitial flogosis and arterial microthrombi (hematoxylin and eosin staining, 40× magnification).
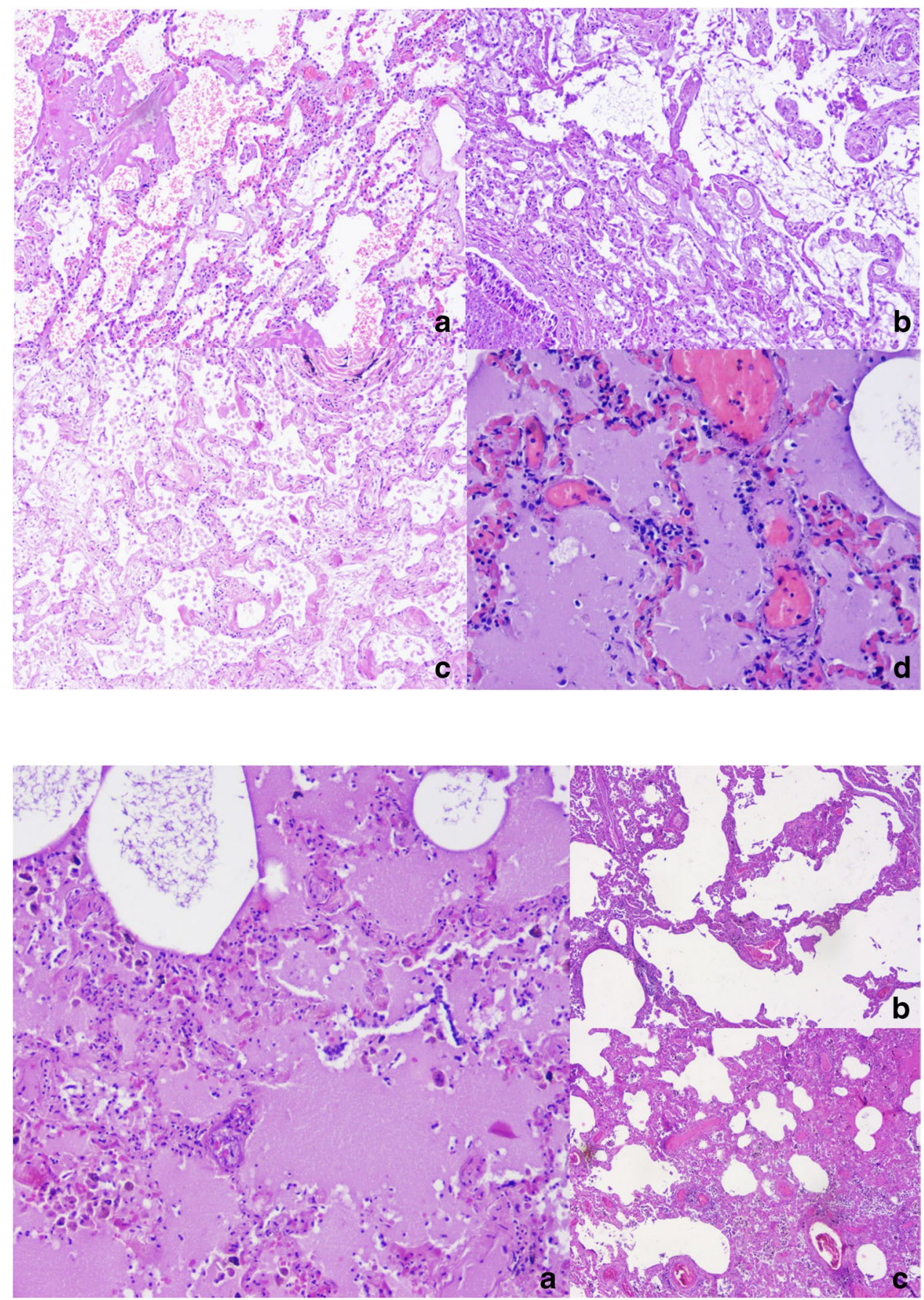

Fig. 5 Histological findings associated with PMCT pattern 3: (a) oedema (hematoxylin and eosin staining, $20 \times$ magnification); (b and c) few signs of SARS-COV-2 infection represented by scant hyaline membrane and focal leukocyte infiltrate (hematoxylin and eosin staining, $5 \times$ magnification).
1) was here detected $[21,23,24]$. The same pattern 1 , attributed to advanced COVID-19 pneumonitis in the clinical setting [8-15], can be interpreted as a common final common pathway in respiratory illness related to severe lung involvement by the pathogen, resembling adult respiratory distress syndrome (ARDS).

This slight difference might be explained by the different study populations, reflecting different stages of COVID19 affecting the lungs, with diffuse consolidations in both lungs being similar to ARDS considered in the clinical and postmortem literature as the expression of more advanced stages of lung pathology due to SARS-CoV-2 infection [14, 21, 23, 24]. Pattern 2 of this study (represented by bilateral and multifocal consolidations and GGO with a "crazy-paving pattern"), similar to other PMCT studies, is the most typical imaging alteration of patients who died by the second week after symptom onset [31]. Finally, in accordance with previous evidence, pattern 3 (constituted by unilateral, eventually multifocal ground-glass opacities) is typical of early stages of COVID-19 [31]. However, none 
of these pulmonary PMCT findings is pathognomonic or has been reported in other infective and noninfective diseases [32-35]. Moreover, in early SARS-CoV-2 lung involvement, the scant and often unilateral GGOs related to COVID-19 might be masked by the above-described classical postmortem changes (gradient densities in dependent lung parenchyma due to hypostasis) or even by superimposed different lung pathologies, such as edema [27] (Fig. 3).

We also found a correlation between progression in the severity of pulmonary alterations on PMCT imaging of the lungs and the severity of pulmonary alterations on histopathological analysis. Several papers in the literature have reported histopathologic findings of lung pathology related to COVID-19 [36-40]. In particular, Buja et al. [41] described the three phases of COVID-19 as follows: acute/ exudative, organizing/proliferative, proliferative/fibrotic. The acute/exudative phase involves intra-alveolar edema and interstitial widening after day 2, possibly associated with diffuse and even focal hyaline membranes. In some cases, it is possible to even note the presence of thrombosis due to alteration of the coagulation pathway. In the organizing phase, cellular fibroblastic proliferation occurs, with type 2 pneumocyte hyperplasia and squamous metaplasia. Hyaline membranes, which appear in the acute phase, start to disappear and become integrated into the alveolar septa. In this phase, it is possible to detect residual fibrin. The end stage of DAD is variable and may be characterized by diffuse fibrosis. According to current evidence, the main COVID19-related histopathological feature is diffuse alveolar damage (DAD), the extent of which varies depending on the stage and severity of the disease. DAD may be associated with intra-alveolar exudates, hyaline membrane, pneumocyte hyperplasia/atypia, alveolar edema, and proteinaceous exudates, with major inflammatory infiltration [36, 37, 40], possible consolidation with fibroblastic proliferation [38, 42], and/or pulmonary thromboemboli [39, 43]. A recent study by Romanova et al. [44] reported that for the majority of cadavers (91\%) positive for SARS-CoV-2 infection whereby autopsy determined cause of death to be other than COVID-19, histopathological analysis of the lungs did not show DAD.

In our study, histopathological examination showed a proliferative phase in $6 / 8$ cases with patterns 1 and 2 on PMCT, corresponding exactly to a more severe pattern of COVID-19 lung disease. On the other hand, cases 7 and 8 exhibited scant signs of infection, as found in the early stage/ acute exudative phase, corresponding to scant antideclive peripheral GGO opacities interpreted as initial pulmonary SARS-CoV-2 involvement on PMCT (pattern 3).

These findings are in accordance with a recently published study aiming to describe the chronology of lung histopathologic changes in COVID-19 and to correlate them with antemortem computed tomography patterns [45]. In this previous study [45], histologic patterns and tomography categories were associated as follows: early/exudative phase correlated with predominantly scant and unilateral GGO, $\mathrm{mid} /$ proliferative phase with crazy paving, and late/fibrous phase correlated with a predominantly consolidation pattern, more frequent in the lower/middle lobes. In our study, fibrosis was found in 3/6 cases with patterns 1 and 2. In accordance with Barisione et al. [45], the detection of fibrosis in our study population might be explained by the duration of mechanical ventilation or oxygen therapy.

Regarding cause of death, in the $6 / 8$ cases with patterns 1 and 2, the cause of death was cardiorespiratory failure due to SARS-CoV-2 infection. In the two cases with pattern 3, with scant signs of SARS-CoV-2 infection in the lungs by both imaging and histological analyses, the cause of death was ischemic heart failure. As a consequence, correlation between the severity of pulmonary PMCT alterations attributed to COVID-19 and the final cause of death might be considered.

This study has several limitations. Undoubtedly, the paucity of the cases strongly limits our results.

Moreover, as indicated above, lung PMCT alterations attributed to COVID-19 are not specific and might be masked or enhanced by postmortem pulmonary changes (hypostasis). Nonetheless, the predominant pattern in fatal COVID-19 cases has been demonstrated in this and previous studies $[18,22]$ to comprise massive GGOs and consolidations in both lungs, a pattern clearly distinguishable based on PMCT findings of pulmonary postmortem changes. Furthermore, the PMCT pattern evidenced in this study might have been the result not only of DAD caused by SARS-CoV-2 but also of the therapy administered during hospitalization before death, such as oxygen therapy and invasive or noninvasive mechanical ventilation.

\section{Conclusion}

Autopsy and histopathological data related to SARSCoV-2 infection remain crucial tools and the gold standard for the study of cadavers positive for SARS-CoV-2 infection. PMCT can be considered a viable screening method for SARS-CoV-2 infection in cadavers [16-22, 24, 46]. Moreover, according to our data, PMCT imaging of the lungs in SARS-CoV-2-positive cadavers correlates well with the severity of lung pathology due to SARS-CoV-2 infection evaluated by histopathological analysis. This suggests that PMCT imaging of the lungs might serve as a reliable method to attribute cause of death to fatal pulmonary COVID-19. Although PMCT alone cannot guarantee a certain diagnosis of COVID-19 as cause of death, our data confirm that it is an extremely important tool for 
causal inference in forensic cases, especially when clinical and microbiological data are also available.

Acknowledgements We are thankful for the valuable technical support of Domenico Laino and Davide Rinelli in performing autopsies.

Funding Funds were from Linea D1, Università Cattolica del Sacro Cuore (recipient: A.O.).

Data availability Data are available on reasonable request to the corresponding author.

\section{Declarations}

Ethics approval The described procedures were authorized by the competing authorities and in accordance with the 1964 Helsinki Declaration and its later amendments.

Consent to participate, consent for publication Consent to participate was not required because the described procedures were authorized by the competing authorities. Consent for publication was not required because applicable law (EU GDPR) does not require consent for publication in scientific research when-as in this case-the manuscript does not contain data referred to identifiable specific individuals.

Competing interests The authors declare no competing interests.

\section{References}

1. Zou L, Ruan F, Huang M et al (2020) SARS-CoV-2 Viral load in upper respiratory specimens of infected patients. New Eng $\mathrm{J}$ Med 382:1177-1179. https://doi.org/10.1056/NEJMc2001737

2. Vasquez-Bonilla WO, Orozco R, Argueta V, Sierra M, Zambrano LI, Muñoz-Lara F, López-Molina DS, Arteaga-Livias K, Grimes Z, Bryce C, Paniz-Mondolfi A, Rodríguez-Morales AJ (2020) A review of the main histopathological findings in coronavirus disease 2019. Hum Pathol 105:74-83

3. Wichmann D (2020) Autopsy findings and venous thromboembolism in patients with COVID-19. Ann Intern Med 173(12):1030. https://doi.org/10.7326/L20-1206

4. Kirschenbaum D, Imbach LL, Rushing EJ, Frauenknecht KBM, Gascho D, Ineichen BV, Keller E, Kohler S, Lichtblau M, Reimann RR, Schreib K, Ulrich S, Steiger P, Aguzzi A, Frontzek $\mathrm{K}$ (2021) Intracerebral endotheliitis and microbleeds are neuropathological features of COVID-19. Neuropathol Appl Neurobiol 47(3):454-459

5. Maiese A, Manetti AC, La Russa R, Di Paolo M, Turillazzi E, Frati P, Fineschi V (2021) Autopsy findings in COVID19-related deaths: a literature review. Forensic Sci Med Pathol 17(2):279-296

6. Xu Z, Shi L, Wang Y et al (2020) Pathological findings of COVID-19 associated with acute respiratory distress syndrome. The Lancet respiratory medicine 8:420-2

7. Kim H, Hong H, Ho YS (2020) Diagnostic performance of ct and reverse transcriptase polymerase chain reaction for coronavirus disease 2019: a meta-analysis. Radiology 296:E145-E155

8. Chung M, Bernheim A, Mei X et al (2020) CT imaging features of 2019 novel coronavirus (2019-nCoV). Radiol 295(1):202207. https://doi.org/10.1148/radiol.2020200230
9. Kong W, Agarwal P (2020) Chest imaging appearance of COVID-19 infection. Radiol Cardiothoracic Imaging. https:// doi.org/10.1148/ryct.2020200028

10. Bernheim A, Mei X, Huang M et al (2020) Chest CT findings in coronavirus disease-19 (COVID-19): relationship to duration of infection. Radiology 259:200463. https://doi.org/10.1148/ radiol.2020200463

11. Pan F, Ye T, Sun P et al (2020) Time course of lung changes on chest CT during recovery from 2019 novel coronavirus (COVID-19) pneumonia. Radiol 295:200370. https://doi.org/ 10.1148/radiol.2020200370

12. Bai HX, Hsieh B, Xiong $Z$ et al (2020) Performance of radiologists in differentiating COVID-19 from viral pneumonia on chest CT. Radiology 296:200823. https://doi.org/10.1148/radiol. 2020200823

13. Salehi S, Abedi A, Balakrishnan S et al (2020) Coronavirus disease 2019 (COVID-19): a systematic review of imaging findings in 919 patients. AJR Am J Roentgenol 215:1-7. https://doi.org/ 10.2214/AJR.20.23034

14. Karimian M, Azami M (2021) Chest computed tomography scan findings of coronavirus disease 2019 (COVID-19) patients: a comprehensive systematic review and meta-analysis. Pol J Radiol. 86:e31-e49. https://doi.org/10.5114/pjr.2021.103379

15. Zhou X, Pu Y, Zhang D, Xia Y, Guan Y, Liu S, Fan L (2021) CT findings and dynamic imaging changes of COVID-19 in 2908 patients: a systematic review and meta-analysis. Acta Radiol 63:291-310. https://doi.org/10.1177/0284185121992655

16. Schweitzer W, Ruder T, Baumeister R et al (2020) Implications for forensic death investigations from first Swiss postmortem CT in a case of non-hospital treatment with COVID-19. Forensic Imaging 21:200378. https://doi.org/10.1016/j.fri.2020. 200378

17. Kniep I, Heinemann A, Edler C, Sperhake JP, Püschel K, Ondruschka B, Schröder AS 2021 COVID-19 lungs in post-mortem computed tomography. Rechts (Berl) 1-3. 15 https://doi.org/10. 1007/s00194-021-00462-z

18. Ducloyer M, Gaborit B, Toquet C et al (2020) Complete postmortem data in a fatal case of COVID-19: clinical, radiological and pathological correlations. Int J Legal Med 134(6):2209-2214. https://doi.org/10.1007/s00414-020-02390-1

19. Helmrich E, Decker L, Adolphi N, Makino Y (2020) Postmortem CT lung findings in decedents with Covid-19: a review of 14 decedents and potential triage implications. Foren Imag 23:200419. https://doi.org/10.1016/j.fri.2020.200419

20. Fitzek A, Sperhake J, Edler C, Schröder AS, Heinemann A, Heinrich F, Ron A, Mushumba H, Lütgehetmann M, Püschel K (2020) Evidence for systematic autopsies in COVID-19 positive deceased: case report of the first German investigated COVID19 death. Rechtsmedizin (Berl) 25:1-6. https://doi.org/10.1007/ s00194-020-00401-4

21. De-Giorgio F, Cittadini F, Cina A, Cavarretta E, Biondi-Zoccai G, Vetrugno G, Natale L, Colosimo C, Pascali VL (2021) Use of post-mortem chest computed tomography in Covid-19 pneumonia. Forensic Sci Int 27(325):110851. https://doi.org/10.1016/j.forsc iint.2021.110851

22. Williams AS, Dmetrichuk JM, Kim P, Pollanen MS (2021) Postmortem radiologic and pathologic findings in COVID-19: the Toronto experience with pre-hospitalization deaths in the community. Forensic Sci Int 322:110755. https://doi.org/10.1016/j. forsciint.2021.110755

23. Filograna L, Manenti G, Arena V, Dell'Aquila M, Pascali VL, Natale L, Colosimo C, Grassi S, Floris R, Oliva A (2021) Claimed medical malpractice in fatal SARS-CoV-2 infections: the importance of combining ante- and post-mortem radiological data and autopsy findings for correct forensic analysis. Forensic Imaging 25:200454. https://doi.org/10.1016/j.fri.2021.200454 
24. O’Donnell C, Iles L, Woodford N (2021) Post-mortem CT lung findings at a medicolegal institute in SARS-CoV-2 RTPCR positive cases with autopsy correlation. Forensic science, medicine, and pathology 17:1-10. https://doi.org/10.1007/ s12024-021-00389-7

25. Malizia A, Filograna L, Ryan CP, Manenti G (2020) Post-mortem investigation through virtual autopsy techniques: proposal of a new diagnostic approach to reduce the risks of operators during emergencies. Int J of Safet and Sec Engin 10(4):535-541

26. Egger C, Vaucher P, Doenz F, Palmiere C, Mangin P, Grabherr S (2012) Development and validation of a postmortem radiological alteration index: the RA-Index. Int J Legal Med 126(4):559-66. https://doi.org/10.1007/s00414-012-0686-6

27. Filograna L, Thali MJ (2017) Post-mortem CT imaging of the lungs: pathological versus non-pathological findings. Radiol Med 122(12):902-908. https://doi.org/10.1007/s11547-017-0802-2

28. Filograna L, Bolliger SA, Ross SG, Ruder T, Thali MJ (2021) Pros and cons of post-mortem CT imaging on aspiration diagnosis. Leg Med (Tokyo) 13(1):16-21. https://doi.org/10.1016/j.legal med.2010.09.006

29. Filograna L, Ross S, Bolliger S, Germerott T, Preiss U, Flach PM, Thali M (2021) Blood aspiration as a vital sign detected by postmortem computed tomography imaging. J Forensic Sci 56(3):630-7. https://doi.org/10.1111/j.1556-4029.2011.01704.x

30. Filograna L, Tartaglione T, Vetrugno G et al (2015) Freshwater drowning in a child: a case study demonstrating the role of post-mortem computed tomography. Med Sci Law 55(4):304-11. https://doi.org/10.1177/0025802414568045

31. Shi H, Han X, Jiang N, Cao Y, Alwalid O, Gu J, Fan Y, Zheng C (2020) Radiological findings from 81 patients with COVID-19 pneumonia in Wuhan China: a descriptive study. Lancet Infect Dis 20(4):425-434. https://doi.org/10.1016/S1473-3099(20)30086-4

32. Wong KT, Antonio GE, Hui DS et al (2004) Severe acute respiratory syndrome: thin-section computed tomography features, temporal changes, and clinicoradiologic correlation during the convalescent period. J Comput Assist Tomogr 28:790-795

33. Qureshi NR, Hien TT, Farrar J et al (2006) The radiologic manifestations of $\mathrm{H} 5 \mathrm{~N} 1$ avian influenza. Journal of thoracic imaging. J Thorac Imaging 21:259-264

34. Paul NS, Roberts H, Butany J et al (2004) Radiologic pattern of disease in patients with severe acute respiratory syndrome: the Toronto experience. Radiographics 24:553-563

35. Das KM, Lee EY, Langer RD et al (2016) Middle east respiratory syndrome coronavirus: what does a radiologist need to know? AJR Am J Roentgenol 206:1193-1201

36. Xu Z, Shi L, Wang Y, Zhang J, Huang L, Zhang C, Liu S, Zhu L, Tai Y, Bai C, Gao T, Song J, Xia P, Dong J, Zhao J, Wang FS (2020) Pathological findings of COVID-19 associated with acute respiratory distress syndrome. Lancet Respir Med 8:420-422. https://doi.org/10.1016/S2213-2600(20)30076-X

37. Tian S, Hu W, Niu L, Liu H, Xu H, Xiao SY (2020) Pulmonary pathology of early-phase 2019 novel coronavirus (COVID-19) pneumonia in two patients with lung cancer. J Thorac Oncol 15:700-704. https://doi.org/10.1016/j.jtho.2020.02.010

38. Menter T, Haslbauer JD, Nienhold R, Savic S, Hopfer H, Deigendesch N, Frank S, Turek D, Willi N, Pargger H, Bassetti S, Leuppi JD, Cathomas G, Tolnay M, Mertz KD, Tzankov A (2020)
Post-mortemexamination of COVID19 patients reveals diffuse alveolar damage with severe capillary congestion and variegated findings of lungs and other organs suggesting vascular dysfunction. Histopathol 77:198-209. https://doi.org/10.1111/his.14134

39. Wichmann D, Sperhake JP, Lütgehetmann M, Steurer S, Edler C, Heinemann A, Heinrich F, Mushumba H, Kniep I, Schröder AS, Burdelski C, de Heer G, Nierhaus A, Frings D, Pfefferle S, Becker $\mathrm{H}$, Bredereke-Wiedling H, de Weerth A, Paschen HR, Sheikhzadeh-Eggers S, Stang A, Schmiedel S, Bokemeyer C, Addo MM, Aepfelbacher M, Püschel K, Kluge S (2020) Autopsy findings and venous thromboembolism in patientswith COVID-19: a prospective cohort study. Ann Intern Med 173:268-277. https://doi.org/ 10.7326/M20-2003

40. Carsana L, Sonzogni A, Nasr A, Rossi RS, Pellegrinelli A, Zerbi P, Rech R, Colombo R, Antinori S, Corbellino M, Galli M, Catena E, Tosoni A, Gianatti A, Nebuloni M (2020) Pulmonary post-mortem findings in a series of COVID-19 cases from northern Italy: a twocentre descriptive study. Lancet Infect Dis 20:1135-1140. https://doi.org/10.1016/S1473-3099(20)30434-5

41. Buja LM, Wolf DA, Zhao B et al (2020) The emerging spectrum of cardiopulmonary pathology of the coronavirus disease 2019 (COVID-19): report of 3 autopsies from Houston, Texas, and review of autopsy findings from other United States cities. Cardiovascular Pathology 48:107233. https://doi.org/10.1016/j. carpath.2020.107233

42. Schaller T, Hirschbühl K, Burkhardt K, Braun G, Trepel M, Märk1 B, Claus R (2020) Postmortem examination of patients with COVID-19. JAMA 323:2518-2520. https://doi.org/10.1001/ jama.2020.8907

43. Dolhnikoff M, Duarte-Neto AN, de Almeida Monteiro RA, Ferraz da Silva LF, Pierre de Oliveira E, Nascimento Saldiva PH, Mauad T, Marcia Negri E (2020) Pathological evidence of pulmonary thrombotic phenomena in severe COVID-19. J Thromb Haemost 18:1517-1519. https://doi.org/10.1111/jth.14844

44. Romanova ES, Vasilyev VV, Startseva G, Karev V, Rybakova MG, Platonov PG (2021) Cause of death based on systematic postmortem studies in patients with positive SARS-CoV-2 tissue PCR during the COVID-19 pandemic. J Intern Med 290:19. https://doi. org/10.1111/joim. 13300

45. Barisione E, Grillo F, Ball L, Bianchi R, Grosso M, Morbini P, Pelosi P, Patroniti NA, De Lucia A, Orengo G, Gratarola A, Verda M, Cittadini G, Mastracci L, Fiocca R (2021) Fibrotic progression and radiologic correlation in matched lung samples from COVID19 post-mortems. Virchows Arch. 478(3):471-485. https://doi. org/10.1007/s00428-020-02934-1

46. Aquila I, Sacco MA, Abenavoli L, Malara N, Arena V, Grassi S, Ausania F, Boccuto L, Ricci C, Gratteri S, Oliva A, Ricci P (2020) Severe acute respiratory syndrome coronavirus 2 pandemic. Arch Pathol Lab Med 144(9):1048-1056. https://doi.org/10.5858/arpa. 2020-0165-SA

Publisher's note Springer Nature remains neutral with regard to jurisdictional claims in published maps and institutional affiliations. 